\title{
Polarization loss correction derived from hydrogen local-resistance measurement in low Pt-loaded polymer-electrolyte fuel cells
}

\author{
Anna T.S. Freiberg ${ }^{\mathrm{a}, \mathrm{b}}$, Michael C. Tucker ${ }^{\mathrm{a}}$, Adam Z. Weber ${ }^{\mathrm{a}, *}$ \\ a Lawrence Berkeley National Laboratory, Energy Technologies Area, 1 Cyclotron Rd, MS 70-108b, Berkeley, CA 94720, United States \\ b Chair of Technical Electrochemistry, Department of Chemistry and Catalysis Research Center, Technische Universität München, D-85748 Garching, Germany
}

\section{A R T I C L E I N F O}

\section{Keywords:}

Hydrogen fuel cell

PEFC

Low Pt-loading

Local resistance

Polarization loss

\begin{abstract}
A B S T R A C T
The reduction of platinum-loading on the cathode side of polymer-electrolyte fuel cells leads to a poorly understood increase in mass-transport resistance (MTR) at high current densities. This local resistance was measured using a facile hydrogen-pump technique with dilute active gases for membrane-electrode assemblies with catalyst layers of varying platinum-loading $\left(0.03-0.40 \mathrm{mg}_{\mathrm{Pt}} / \mathrm{cm}^{2}\right)$. Furthermore, polarization curves in $\mathrm{H}_{2} /$ air were measured and corrected for the overpotential caused by the increased MTR for low loadings on the air side due to the reduced concentration of reactant gas at the catalyst surface. The difference in performance after correction for all resistances including the MTR is minor, suggesting its origin to be diffusive in nature, and proving the meaningfulness of the facile hydrogen-pump technique for the characterization of the cathode catalyst layer under defined operation conditions.
\end{abstract}

\section{Introduction}

Polymer-electrolyte fuel cells (PEFCs) are promising candidates as energy-efficient power sources for transportation applications due to their outstanding volumetric and gravimetric power and energy densities compared to state-of-the-art lithium-ion batteries. However, their cost is still prohibitive, and thus there is a strong need to reduce the expensive platinum catalyst loadings. A target of $0.125 \mathrm{~g}_{\mathrm{PGM}} / \mathrm{kW}$ was set by the U.S. Department of Energy by the year 2020 [1]. Regrettably already for loadings below $0.10 \mathrm{mg} \mathrm{P}_{\mathrm{Pt}} / \mathrm{cm}^{2}$ at the cathode side, the performance suffers quite drastically due to a poorly understood local resistance, which is thought to be related to an increased local mass-transport resistance (MTR), although other causes have been proposed as well [2-13]. In terms of physical interpretation, the MTR represents the local resistance of a reactant molecule towards reaching an active reaction site, which becomes more significant as the number of reaction sites decrease but the desired overall reaction rate remains the same.

The concept of the local MTR has gained acceptance as several studies seem to suggest that there could be such a resistance through the ionomer thin-film or at the interface of ionomer and electrocatalyst, and which is linearly related with the inverse of the roughness factor ( $r f$ ). Based on an agglomerate model, Greszler et al. [2] have concluded that this resistance cannot be explained by pure geometrical inhomogeneity, which was reviewed in detail from an ionomer point of view by
Weber and Kusoglu [3]. It is believed that this resistance is due to an intrinsic property of the ionomer thin-film, which differs from the bulk property and/or the interfacial resistance at the $\mathrm{Pt} /$ ionomer interface [2-4,7,10,14-19]. As imaging of the ionomer and polymer based analysis/spectroscopy is difficult and mostly only possible ex situ, the direct quantification of this resistance in situ or operando is of high interest, especially for fast characterization of the various membraneelectrode assemblies (MEAs) comprised of membrane and anode and cathode catalyst layers (CLs) [4].

The MTR can be measured by limiting current as it is related primarily to local mass transport. The traditional technique of oxygen limiting-current makes this complicated by various factors including concomitant water production, possible existence of surface oxides, as well as heat generation and related effects due to the inefficiency of the oxygen-reduction-reaction, etc. [8] Recently, a limiting-current based on a hydrogen-pump setup has been tried, which alleviates many of the issues with respect to oxygen limiting-current, but the direct applicability of the measurement towards PEFCs under operation is unknown [20]. In this study, we examine whether the MTR by hydrogen limitingcurrent experiments can be correlated to the overall performance loss in $\mathrm{H}_{2}$ /air polarization curves, thereby affirming that diffusion or massdependent processes govern the observed performance losses with low Pt-loadings.

\footnotetext{
* Corresponding author.

E-mail address: azweber@lbl.gov (A.Z. Weber).
} 
Table 1

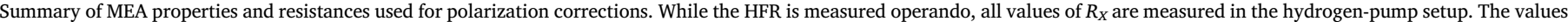

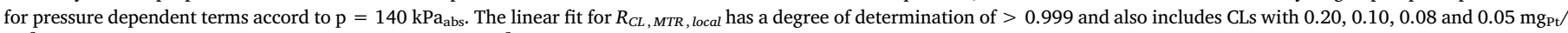
$\mathrm{cm}^{2}$. Please note that in case of the CL with $0.40 \mathrm{mg} \mathrm{Pt}_{\mathrm{Pt}} / \mathrm{cm}^{2}$ the carbon based catalyst used, bears a higher Pt-loading, which leads to the thinner CL.

\begin{tabular}{|c|c|c|c|c|c|c|c|c|}
\hline \multirow[b]{2}{*}{ WE loading $\left[\mathrm{mg}_{\mathrm{Pt}} / \mathrm{cm}^{2}\right]$} & \multirow[b]{2}{*}{$\mathrm{t}_{\mathrm{CL}}[\mu \mathrm{m}]$} & \multicolumn{3}{|c|}{ Ohmic resistances } & \multicolumn{4}{|c|}{ Mass-transport resistances } \\
\hline & & $\mathrm{HFR}\left[\mathrm{m} \Omega \mathrm{cm}^{2}\right]$ & $R_{\text {sheet }, C E}\left[\mathrm{~m} \Omega \mathrm{cm}^{2}\right]$ & $R_{\text {sheet }, W E}\left[\mathrm{~m} \Omega \mathrm{cm}^{2}\right]$ & $R_{\text {total }, H_{2}}[\mathrm{~s} / \mathrm{m}]$ & $R_{G D L}[\mathrm{~s} / \mathrm{m}]$ & $\operatorname{rf}[-]$ & $R_{C L . M T R, \text { local }}[\mathrm{s} / \mathrm{m}]$ \\
\hline 0.03 & 2.8 & 68 & 664 & 152 & $32.26 \pm 0.89$ & 10.40 & 19.46 & 314.72 \\
\hline 0.15 & 14 & 52 & & 935 & $14.10 \pm 0.07$ & & 123.25 & \\
\hline 0.40 & 8 & 54 & & 664 & $12.97 \pm 0.15$ & & 243.87 & \\
\hline
\end{tabular}

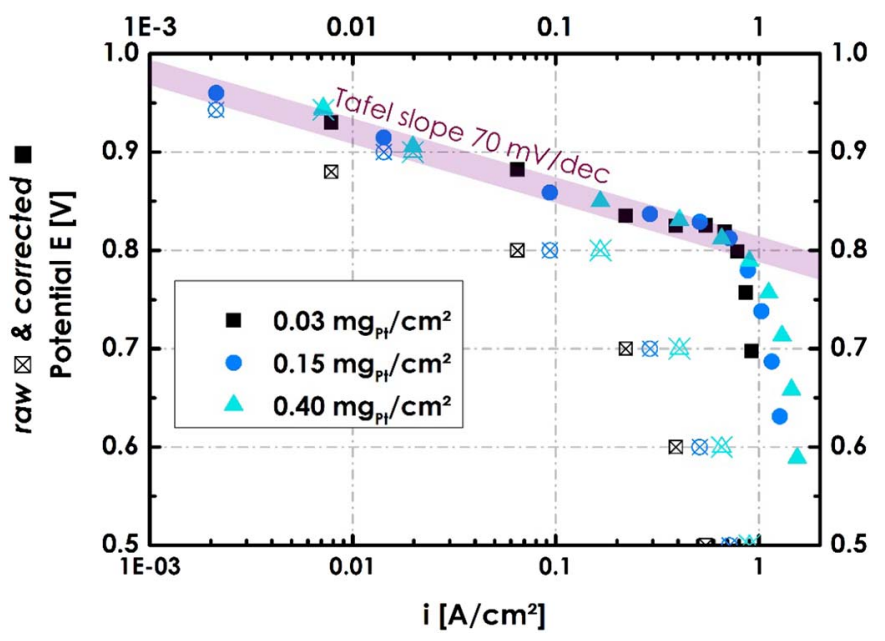

Fig. 1. Polarization curves of MEAs with varying WE platinum-loading at $80{ }^{\circ} \mathrm{C}, 90 \% \mathrm{RH}$ in $\mathrm{H}_{2}$ /air at $140 \mathrm{kPa}_{\text {abs. }}$. The crossed symbols represent the raw data, filled symbols show the same data set after iR- and MTR-correction, which collapse the data; a Tafel slope of $70 \mathrm{mV} / \mathrm{dec}$ is added as a guide to the eye.

\section{Theory}

If the MTR is dominated by a traditional diffusion-like masstransport step, one expects that the resistance, $R_{M T R}$, which is inversely related to the diffusion coefficient, $D$, will vary as a square root dependence on molecular weight, $\mathrm{M}$, of the diffusing gas,

$\frac{1}{R_{M T R}} \propto D \propto \frac{1}{\sqrt{M}}$

Even though the hydrogen limiting-current experiments were executed in a gas mixture containing $1000 \mathrm{ppm} \mathrm{H}_{2}$ in $\mathrm{Ar}$, it is assumed that unimolecular diffusion of hydrogen dominates and one can determine the total hydrogen-gas resistance, $R_{\text {total } \mathrm{H}_{2}}$, using a Fickianderived expression

$R_{\text {total }, H_{2}}=\frac{2 \cdot F \cdot c_{H_{2}, \text { bulk }}}{i_{\text {lim }}}$

where $i_{\text {lim }}$ is the limiting-current density, $F$ is the Faraday's constant, and $c_{H_{2}, \text { bulk }}$ is the concentration of hydrogen in the flow field, which is calculated based on the ideal-gas law using the universal gas constant $\bar{R}$ and the molar flow of hydrogen fed $\dot{n}_{H_{2} \text { feed }}$ and consumed $\dot{n}_{H_{2} \text {,lim }}$

$c_{H_{2}, \text { bulk }}=\frac{p}{\bar{R} \cdot T} \cdot \frac{\dot{n}_{H_{2}, \text { feed }}-\dot{n}_{H_{2}, \text { lim }}}{\dot{n}_{H_{2}, \text { feed }}}$

The measured mass-transport resistance for hydrogen $R_{\text {total, } H_{2}}$ can be divided up into 3 portions assuming resistances in series for the gas species to reach the catalyst site [2]

$R_{\text {total }, H_{2}}=n \bullet R_{G D L}+R_{C L, p}+\frac{1}{r f} \cdot R_{C L, M T R, \text { local }}$

where $R_{G D L}$ corresponds to the pressure dependent resistance in the gas diffusion layer (GDL). This resistance includes both Knudsen and molecular diffusion within the pores of the GDL. $\mathrm{n}$ corresponds to the numbers of stacked GDLs, $R_{C L, p}$ refers to the pressure dependent portion of the MTR of the CL within the pores (including both Knudsen and molecular diffusion), $r f$ is the measured catalyst roughness factor $\left(\mathrm{cm}_{\mathrm{Pt}}^{2} /\right.$ $\mathrm{cm}^{2}$ geometric $)$ that depends on loading, and $R_{C L, M T R, \text { local }}$ accounts for the local MTR, which is nominally loading independent. This additional resistance is seen to be caused by the ionomer thin-film and/or its interface with the catalyst. The interfacial resistance of the ionomer towards the gas-phase is not explicitly incorporated herein which was chosen as the sorption resistance at elevated temperatures and relative humidity (RH) is minor compared to the local MTR close to the catalyst surface and is therefore presumed to have no impact on the correlation of $\mathrm{H}_{2}$-based MTR evaluation and the corresponding polarization performance in the $\mathrm{H}_{2}$ /air set-up.

The reduced reactant concentration over the catalyst leads to an increasing overpotential at high current densities $\Delta \eta_{M T R}$. MTR induced losses are calculated based on eq. (5) which is derived from Nernst' equation as shown by Zihrul et al. [21]

$\Delta \eta_{M T R}=\frac{\bar{R} \cdot T}{F} \cdot\left(\frac{1}{4}+\frac{\gamma}{\alpha}\right) \cdot \ln \left(\frac{p_{O_{2}, \text { channel }}-\frac{\bar{R} T}{4 F} \cdot R_{\text {total }, O_{2}} \bullet i}{p_{O_{2}, \text { channel }}}\right)$

The bracket on the right represents the ratio of reactant gas at the catalyst surface to provided reactant $p_{\mathrm{O}_{2}}$, channel. $\alpha$ and $\gamma$ are the transfer coefficient and oxygen-reduction-reaction reaction order, respectively; the values from Zihrul et al. [21] are taken for the calculations herein ( 1 and 0.54 , respectively).

Assuming that the mass-transport is a diffusion process without any noticeable sorption effects, the ratio of MTR for hydrogen and oxygen corresponds to the ratio of the square root of their molecular-masses $\left(\frac{\sqrt{32}}{\sqrt{2}}=4\right)$ leading to

$\Delta \eta_{M T R}=\frac{\bar{R} \cdot T}{F} \cdot\left(\frac{1}{4}+\frac{\gamma}{\alpha}\right) \cdot \ln \left(\frac{p_{O_{2}, \text { channel }}-\frac{\bar{R} T}{F} \bullet R_{\text {total }, H_{2}} \bullet i}{p_{O_{2}, \text { channel }}}\right)$

This correlation between the $\mathrm{H}_{2}$-based MTR and the oxygen MTR is perhaps not valid for interfacial resistances as sorption effects unless they follow the same mass dependence. As shown later, the assumption of a diffusion process being the cause of the increased MTR holds true, which makes eq. 6 a feasible approximation of the overpotential caused by the MTR.

Besides the correction for the mass-transport loss, already established iR-corrections used in literature were performed as well to correct for contact and ionic resistances within the membrane and CL ionomer of the working (WE) and counter electrode (CE). Their determination was carried out using the approaches described by Neyerlin et al. [22].

\section{Experimental}

MEAs were fabricated by decal transfer of a CE CL with a loading of $0.40 \mathrm{mg}_{\mathrm{Pt}} / \mathrm{cm}^{2}$ and a varying WE CL with a loading of 0.03 to $0.40 \mathrm{mg}_{\mathrm{Pt}} / \mathrm{cm}^{2}(\mathrm{I} / \mathrm{C}=0.75)$ on a commercial Nafion ${ }^{\circledR}$ NRE212 membrane [23]. The CL was coated out of an ink consisting of DE2020CS ionomer (Dupont) and TEC10E50E $\left(0.40 \mathrm{mg}_{\mathrm{Pt}} / \mathrm{cm}^{2}\right)$ or TEC10E20E 

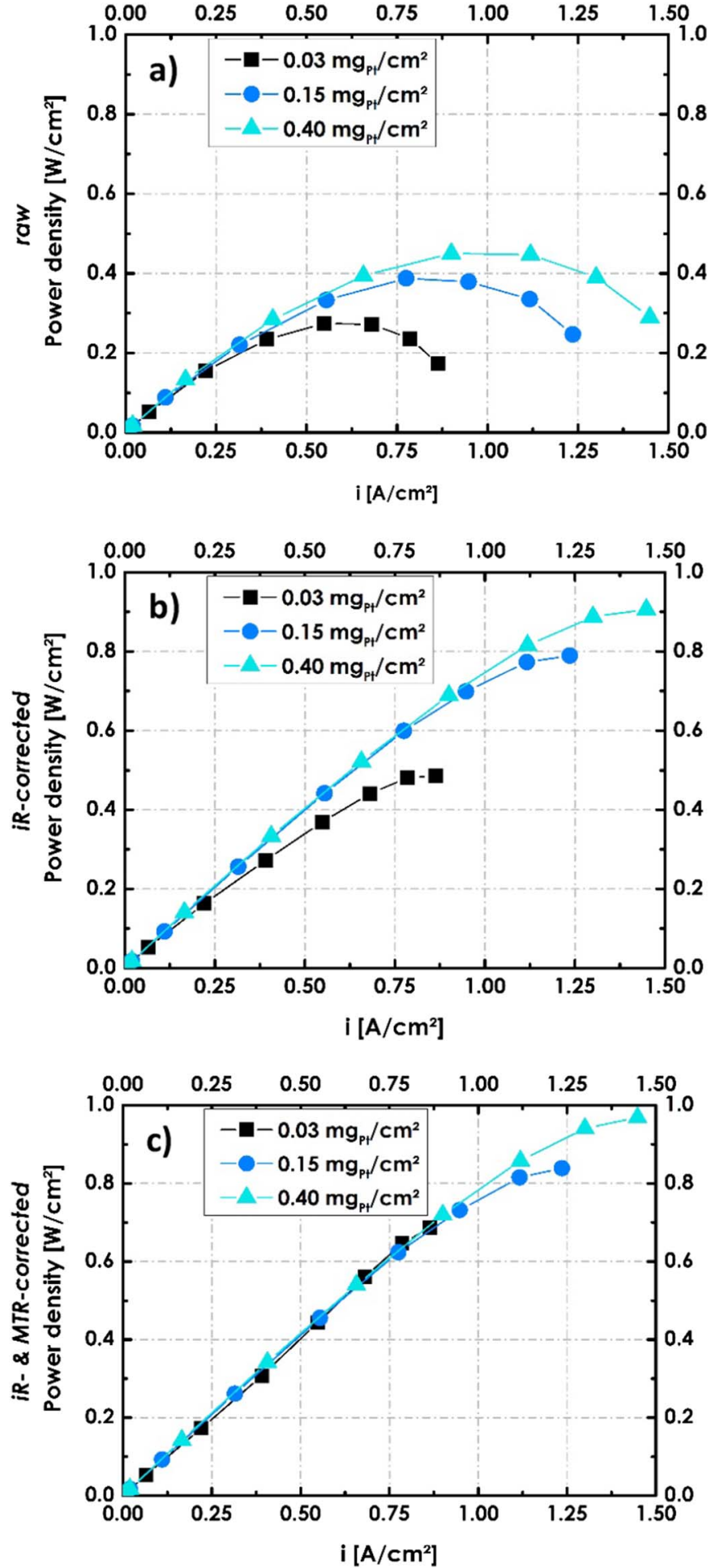

Fig. 2. Power density as a function of current density for different WE Pt-loadings showing (a) raw data, (b) after iR-correction for contact and ionic resistances, and (c) after iR- and MTR-correction. The corresponding polarization curves are shown in Fig. 1.

(0.15 and $0.03 \mathrm{mg}_{\mathrm{Pt}} / \mathrm{cm}^{2}$ ) catalyst powder (Tanaka). The CL roughness factor, $r f$, was calculated based on the electrochemical active surface area (ECSA), which was determined by CO-stripping voltammetry assuming a specific charge density of $q_{C O, P t}=420 \frac{\mu C}{\mathrm{~cm}_{P t}^{2}}$ [24].

Following the work of Spingler et al. [20], testing of the local hydrogen MTR was executed in a $1 \mathrm{~cm}^{2}$ test cell at a test station by Fuel Cell Technologies Inc. using several layers of SGL ${ }^{\circledast}$ Sigracet 24 BA on the WE CL feeding $500 \mathrm{~cm}^{3} / \mathrm{min}$ of $1000 \mathrm{ppm} \mathrm{H}_{2}$ in Ar. To avoid large crossover currents, the CE using only one layer of SGL ${ }^{\circledast}$ Sigracet $24 \mathrm{BA}$ was fed with $200 \mathrm{~cm}^{3} / \mathrm{min}$ of $2 \% \mathrm{H}_{2}$ in Ar providing a stable reference potential. Several GDLs ( 3 to 5 ) were stacked at the WE to subtract the MTR caused by the GDL by a linear-extrapolation method [20]. Limiting-current experiments were executed by measuring the equilibrium current at $0.35 \mathrm{~V}$ at $80{ }^{\circ} \mathrm{C}, 90 \% \mathrm{RH}$ with a backpressure of $107 \mathrm{kPa}_{\mathrm{abs}}$ and $170 \mathrm{kPa}_{\mathrm{abs}}$ to determine the pressure dependent MTR (see reference [20] for more details and cell setup).

Hydrogen/air polarization curves were recorded using a $5 \mathrm{~cm}^{2}$ cell (Fuel Cell Technologies) and a Scribner 850e fuel cell test stand. The voltage was varied between OCV and $0.2 \mathrm{~V}$ in $100 \mathrm{mV}$ increments using purified air and $\mathrm{H}_{2}$ (420 and $200 \mathrm{~cm}^{3} / \mathrm{min}$, respectively) at $140 \mathrm{kPa}_{\mathrm{abs}}$, $80{ }^{\circ} \mathrm{C}$ and $90 \% \mathrm{RH}$. Herein one layer of SGL ${ }^{\circledR}$ Sigracet $25 \mathrm{BC}$ was used as GDL to improve the gas and water distribution. The resistance of the GDL was incorporated into the polarization loss correction of equ. (6) using the value obtained by Spingler et al. [20]. Impedance measurements needed for the iR-corrections following Liu et al. [25] were recorded using a Biologic VSP3 potentiostat at OCV in potentiostatic mode using a perturbation of $5 \mathrm{mV}$.

As the local MTR is measured in a set-up that is significantly differing from the $\mathrm{H}_{2}$ /air polarization set-up in terms of ohmic losses and MTR caused by GDL, a deconvolution of every resistive term is necessary using the methods detailed in the theoretical and experimental part herein.

\section{Results and discussion}

Table 1 summarizes all of the data needed for correction of the polarization curves for the CLs discussed here $(0.40,0.15$, and $0.03 \mathrm{mg}_{\mathrm{Pt}} / \mathrm{cm}^{2}$ ). The high frequency resistance (HFR) is, as expected, roughly the same for all samples. The differences are rather attributed to variance of cell compression due to the different CL thickness than an intrinsic deviation in electronic contact with the CL. The ionic resistance within the CL of the hydrogen side, $R_{\text {sheet }, C E}$ is set to be the constant value of a CL with $0.40 \mathrm{mg}_{\mathrm{Pt}} / \mathrm{cm}^{2}$ measured for a symmetric MEA. The only really varying portion in the iR-correction term is the sheet resistance of the WE CL, $R_{\text {sheet, } W E}$. The sheet resistance represents the proton transport resistance within the CL and was measured in blocking conditions as described in the literature [25-27]. $R_{\text {sheet }} W E$ roughly correlates to the CL thickness, $\mathrm{t}_{\mathrm{CL}}$, which varies due to the overall Pt-loading. The MTR is significantly larger for the $0.03 \mathrm{mg}_{\mathrm{Pt}} /$ $\mathrm{cm}^{2} \mathrm{CL}$ as already reported in literature $[2-8,10,14,20]$.

The $\mathrm{H}_{2}$ /air polarization performance for the 3 MEAs is shown in Fig. 1, where the hollow crossed symbols represent the raw data. It is readily apparent that the performance loss for the low-loaded CL is largely enhanced even at current densities as low as $10 \mathrm{~mA} / \mathrm{cm}^{2}$. The filled symbols represent the same data after iR- and MTR-correction. Now all samples exhibit comparable polarization behavior with only minor deviations. They all follow the kinetic regime until $800 \mathrm{~mA} / \mathrm{cm}^{2}$ and then even the higher loaded CLs show enhanced polarization. As the correction was executed with an oxygen MTR calculated from measured hydrogen MTR, this correction is not expected to result in quantitative agreement in the kinetic regime, but the fact that after correction all of the samples transition at the same current density proves the meaningfulness of the hydrogen MTR for quantification of the impact of Pt-loading. Our local resistance $R_{C L, M T R, \text { local }}$ for hydrogen fits to the local resistance for oxygen reported in literature (minding the factor of 4) [2,4]. The slightly higher hydrogen-based $R_{C L, M T R, \text { local }}$ can be explained by over-heating of the catalyst during standard oxygen limiting-current experiments, which will inevitably lead to an underestimation of the local MTR.

The impact of the correction and applicability of the hydrogenbased MTR is shown further in Fig. 2 comparing the power density curves for the different MEAs. While the raw data (a) show substantial differences, after iR-corrections (b) those differences become small but they still clearly exhibit deviations, even at rather low current densities. 
When comparing the power densities obtained after iR- and MTRcorrection (c), those differences tend to vanish. For the case of the $0.03 \mathrm{mg}_{\mathrm{Pt}} / \mathrm{cm}^{2} \mathrm{CL}$, the MTR-correction leads to a total vanishing of peak performance showing that the decaying arc for high current densities in Fig. 2b is caused solely by a diffusive or at least square root molecular-mass dependent MTR. The deviation of the $0.15 \mathrm{mg} \mathrm{gt}_{\mathrm{Pt}} / \mathrm{cm}^{2}$ $\mathrm{CL}$ at high current densities reveals that the correction is not perfect. It is presumed that at such high current densities the impact of water production on the MTR is becoming significant, which is especially crucial for thick CLs as in case of the $0.15 \mathrm{mg}_{\mathrm{Pt}} / \mathrm{cm}^{2} \mathrm{CL}$. This also highlights the fact that issues such as water and heat production require further study as it impacts performance at the local scale (e.g., waterfilm formation). Nevertheless, the good agreement demonstrates that the correction for MTR is successful.

\section{Conclusion}

The concept of a local resistance leading to enhanced polarization losses in low Pt-loaded polymer-electrolyte fuel cell (PEFC) membraneelectrode assemblies (MEAs) is established in the literature, although the exact cause is unknown. In this article, we have demonstrated that this resistance is a mass-transport resistance (MTR), which follows a diffusion-type mass dependence. Furthermore, it is shown how a facile hydrogen-pump experiment can be easily employed to quantify this effect, thereby ruling out activation processes, water production, oxide effects, possible peroxide formation, and enhanced heat generation during oxygen limiting-current experiments at low potentials. However, such effects do seem to exist at least partially at higher operating current densities. The use of the hydrogen-pump technique allows for rapid characterization of MEAs and further analysis of the limiting steps during PEFC operation. After iR- and "hydrogen-based" MTR-corrections, the remaining resistances in the polarization-curve prediction are due to kinetic limitations (approximated by Tafel-behavior) and operation dependent "water-production-caused" MTR. Future work will need to examine the influence of temperature, humidity and related variables on the MTR, and focus on the optimization of the Pt/ionomer interface and ionomer properties to enhance Pt-utilization and decrease the MTR.

\section{Acknowledgements}

Sincere thanks are given to Dr. Alin Orfanidi and Christoph Simon from the TEC fuel cell group at Technische Universität München as well as Prof. Dr. Marc Secanell from ESDLab at University of Alberta, Edmonton for helpful discussion. This work was conducted under the Fuel Cell Performance and Durability Consortium (FC-PAD), which is supported by the Fuel Cell Technologies Program, Office of Energy Efficiency and Renewable Energy, of the U.S. Department of Energy under Contract No. DE-AC02-05CH11231.

\section{References}

[1] A.Z. Weber, Modeling Water-Management in Polymer-Electrolyte Fuel Cells, in: Department of Chemical Engineering, University of California, Berkeley, 2004.

[2] T.A. Greszler, D. Caulk, P. Sinha, The impact of platinum loading on oxygen transport resistance, J. Electrochem. Soc. 159 (2012) F831-F840.

[3] A.Z. Weber, A. Kusoglu, Unexplained transport resistances for low-loaded fuel-cell catalyst layers, J. Mater. Chem. A 2 (2014) 17207-17211.

[4] A. Kongkanand, M.F. Mathias, The priority and challenge of high-power performance of low-platinum proton-exchange membrane fuel cells, J. Phys. Chem. Lett. 7 (2016) 1127-1137.

[5] Y. Ono, T. Mashio, S. Takaichi, A. Ohma, H. Kanesaka, K. Shinohara, The analysis of performance loss with low platinum loaded cathode catalyst layers, ECS Trans. 28 (2010) 69-78.

[6] N. Nonoyama, S. Okazaki, A.Z. Weber, Y. Ikogi, T. Yoshida, Analysis of oxygentransport diffusion resistance in proton-exchange-membrane fuel cells, $\mathrm{J}$. Electrochem. Soc. 158 (2011) B416-B423.

[7] J.P. Owejan, J.E. Owejan, W. Gu, Impact of platinum loading and catalyst layer structure on PEMFC performance, J. Electrochem. Soc. 160 (2013) F824-F833.

[8] D.R. Baker, D.A. Caulk, K.C. Neyerlin, M.W. Murphy, Measurement of oxygen transport resistance in PEM fuel cells by limiting current methods, J. Electrochem. Soc. 156 (2009) B991-B1003.

[9] M. Breitwieser, M. Klingele, B. Britton, S. Holdcroft, R. Zengerle, S. Thiele, Improved Pt-utilization efficiency of low Pt-loading PEM fuel cell electrodes using direct membrane deposition, Electrochem. Commun. 60 (2015) 168-171.

[10] A. Ohma, T. Mashio, K. Sato, H. Iden, Y. Ono, K. Sakai, K. Akizuki, S. Takaichi, K. Shinohara, Analysis of proton exchange membrane fuel cell catalyst layers for reduction of platinum loading at Nissan, Electrochim. Acta 56 (2011) 10832-10841.

[11] W. Yoon, A.Z. Weber, Modeling low-platinum-loading effects in fuel-cell catalyst layers, J. Electrochem. Soc. 158 (2011) B1007-B1018.

[12] L. Hao, K. Moriyama, W. Gu, C.Y. Wang, Modeling and experimental validation of Pt loading and electrode composition effects in PEM fuel cells, J. Electrochem. Soc. 162 (2015) F854-F867.

[13] A. Kongkanand, N.P. Subramanian, Y. Yu, Z. Liu, H. Igarashi, D.A. Muller, Achieving high-power PEM fuel cell performance with an ultralow-Pt-content core-shell catalyst, ACS Catal. 6 (2016) 1578-1583.

[14] H. Liu, W.K. Epting, S. Litster, Gas transport resistance in polymer electrolyte thin films on oxygen reduction reaction catalysts, Langmuir 31 (2015) 9853-9858.

[15] A. Kusoglu, A.Z. Weber, New insights into perfluorinated sulfonic-acid ionomers, Chem. Rev. 117 (2017) 987-1104.

[16] M.A. Hickner, H. Ghassemi, Y.S. Kim, B.R. Einsla, J.E. McGrath, Alternative polymer systems for Proton Exchange Membranes (PEMs), Chem. Rev. 104 (2004) 4587-4612.

[17] A. Kusoglu, D. Kushner, D.K. Paul, K. Karan, M.A. Hickner, A.Z. Weber, Impact of substrate and processing on confinement of nafion thin films, Adv. Funct. Mater. 24 (2014) 4763-4774.

[18] V.S. Murthi, J. Dura, S. Satija, C. Majkrzak, Water uptake and interfacial structural changes of thin film Nafion ${ }^{\circledast}$ membranes measured by neutron reflectivity for PEM fuel cells, ECS Trans. 16 (2008) 1471-1485.

[19] M.A. Modestino, D.K. Paul, S. Dishari, S.A. Petrina, F.I. Allen, M.A. Hickner, K. Karan, R.A. Segalman, A.Z. Weber, Self-assembly and transport limitations in confined nafion films, Macromolecules 46 (2013) 867-873.

[20] F.B. Spingler, A. Phillips, T. Schuler, M.C. Tucker, A.Z. Weber, Investigating fuelcell transport limitations using hydrogen limiting current, Int. J. Hydrog. Energy (2017), http://dx.doi.org/10.1016/j.ijhydene.2017.01.036.

[21] P. Zihrul, I. Hartung, S. Kirsch, G. Huebner, F. Hasché, H.A. Gasteiger, Voltage cycling induced losses in electrochemically active surface area and in H2/airperformance of PEM fuel cells, J. Electrochem. Soc. 163 (2016) F492-F498.

[22] K.C. Neyerlin, W. Gu, J. Jorne, A. Clark, H.A. Gasteiger, Cathode catalyst utilization for the ORR in a PEMFC, J. Electrochem. Soc. 154 (2007) B279-B287.

[23] M.S. Wilson, S. Gottesfeld, Thin-film catalyst layers for polymer electrolyte fuel cell electrodes, J. Appl. Electrochem. 22 (1992) 1-7.

[24] J. Durst, C. Simon, F. Hasche, H.A. Gasteiger, Hydrogen oxidation and evolution reaction kinetics on carbon supported Pt, Ir, Rh, and Pd electrocatalysts in acidic media, J. Electrochem. Soc. 162 (2014) F190-F203.

[25] Y. Liu, M. Murphy, D. Baker, W. Gu, C. Ji, J. Jorne, H.A. Gasteiger, Determination of electrode sheet resistance in cathode catalyst layer by AC impedance, ECS Trans. 11 (2007) 473-484.

[26] D. Malevich, J. Pharoah, B. Peppley, K. Karan, On the determination of PEM fuel cell catalyst layer resistance from Impedance measurement in H2/N2 cells, J. Electrochem. Soc. 159 (2012) F888-F895.

[27] M. Cimenti, D. Bessarabov, M. Tam, J.R. Stumper, Investigation of proton transport in the catalyst layer of PEM fuel cells by electrochemical impedance spectroscopy, ECS Trans. 28 (2010) 147-157. 